

\title{
Engagement et mobilisation de parents autour de l'école
} Yves Dutercq, Claudette Lafaye

\section{To cite this version:}

Yves Dutercq, Claudette Lafaye. Engagement et mobilisation de parents autour de l'école: Les actions pour le maintien en ZEP d'écoles parisiennes. Revue française de sociologie, 2003, 44 (3), pp.469-495. 10.3917/rfs.443.0469 . hal-01247342

\section{HAL Id: hal-01247342 \\ https://hal.science/hal-01247342}

Submitted on 21 Dec 2015

HAL is a multi-disciplinary open access archive for the deposit and dissemination of scientific research documents, whether they are published or not. The documents may come from teaching and research institutions in France or abroad, or from public or private research centers.
L'archive ouverte pluridisciplinaire HAL, est destinée au dépôt et à la diffusion de documents scientifiques de niveau recherche, publiés ou non, émanant des établissements d'enseignement et de recherche français ou étrangers, des laboratoires publics ou privés. 


\section{ENGAGEMENT ET MOBILISATION DE PARENTS AUTOUR DE L'ÉCOLE}

Les actions pour le maintien en ZEP d'écoles parisiennes

Yves Dutercq, Claudette Lafaye

Presses de Sciences Po (P.F.N.S.P.) | « Revue française de sociologie »

2003/3 Vol. 44 | pages 469 à 495

ISSN 0035-2969

ISBN 2708010573

Article disponible en ligne à l'adresse :

http://www.cairn.info/revue-francaise-de-sociologie-2003-3-page-469.htm

!Pour citer cet article :

Yves Dutercq, Claudette Lafaye, «Engagement et mobilisation de parents autour de l'école. Les actions pour le maintien en ZEP d'écoles parisiennes », Revue française de sociologie 2003/3 (Vol. 44), p. 469-495.

DOI 10.3917/rfs.443.0469

Distribution électronique Cairn.info pour Presses de Sciences Po (P.F.N.S.P.).

(C) Presses de Sciences Po (P.F.N.S.P.). Tous droits réservés pour tous pays.

La reproduction ou représentation de cet article, notamment par photocopie, n'est autorisée que dans les limites des conditions générales d'utilisation du site ou, le cas échéant, des conditions générales de la licence souscrite par votre établissement. Toute autre reproduction ou représentation, en tout ou partie, sous quelque forme et de quelque manière que ce soit, est interdite sauf accord préalable et écrit de l'éditeur, en dehors des cas prévus par la législation en vigueur en France. Il est précisé que son stockage dans une base de données est également interdit. 


\title{
Engagement et mobilisation de parents autour de l'école
}

Les actions pour le maintien en ZEP d'écoles parisiennes

Ophrys | Revue française de sociologie

\author{
2003/3 - Volume 44 \\ pages 469 à 495 \\ ISSN 0035-2969
}

Article disponible en ligne à l'adresse:

http://www.cairn.info/revue-francaise-de-sociologie-2003-3-page-469.htm

Pour citer cet article :

"Engagement et mobilisation de parents autour de l'école" Les actions pour le maintien en ZEP d'écoles parisiennes,

Distribution électronique Cairn.info pour Ophrys.

(C) Ophrys. Tous droits réservés pour tous pays.

La reproduction ou représentation de cet article, notamment par photocopie, n'est autorisée que dans les limites des conditions générales d'utilisation du site ou, le cas échéant, des conditions générales de la licence souscrite par votre établissement. Toute autre reproduction ou représentation, en tout ou partie, sous quelque forme et de quelque manière que ce soit, est interdite sauf accord préalable et écrit de l'éditeur, en dehors des cas prévus par la législation en vigueur en France. II est précisé que son stockage dans une base de données est également interdit. 


\author{
Yves DUTERCQ \\ Claudette LAFAYE
}

\title{
Engagement et mobilisation de parents autour de l'école
}

\author{
Les actions pour le maintien en ZEP d'écoles parisiennes*
}

\begin{abstract}
RÉSUMÉ
L'étude d'un conflit centré sur le maintien d'écoles élémentaires dans le dispositif de zone d'éducation prioritaire (ZEP) permet de clarifier les figures de montée en généralité dans les engagements et les mobilisations autour de l'école. La mobilisation que nous avons analysée en nous fondant sur les récits rétrospectifs de ses différents protagonistes montre la possibilité pour des mouvements locaux de s'articuler à des revendications non particularistes, sans toutefois oublier les motifs initiaux d'insatisfaction. Cet accès à une cause argumentée, au-delà du travail de médiatisation du mouvement, lui a donné sa consistance interne. En effet, à partir d'un ancrage localisé, ces formes d'action ont fait émerger des questions d'intérêt général que nous présentons et discutons. La clôture de la controverse montre cependant que si l'administration accède à la satisfaction de la revendication initiale des parents, elle méconnaît sa légitimité.
\end{abstract}

Si les relations entre l'école et ses usagers ont déjà fait l'objet d'investigations nombreuses, qu'il s'agisse de l'histoire des fédérations de parents d'élèves et de leurs difficultés à constituer une cause (Barthélémy, 1995 ; Dutercq, 1995, 1998) ou des formes récentes de consumérisme scolaire (Ballion, 1982 ; Ashworth, 1991 ; Derouet, 1991 ; Dutercq, 1993), les mobilisations locales de parents ont été quelque peu délaissées. Les rares travaux existant concernent pour l'essentiel le niveau de l'enseignement secondaire (Masson, 1997), sans doute parce que les inquiétudes des parents demeurent majoritairement focalisées sur le collège (qu'il s'agisse de violences, d'incivi-

* Cette recherche n'aurait pu aboutir sans la contribution de Christian Baly, enseignant associé à l'INRP (Groupe d'études sociologiques) qui a réalisé l'essentiel du travail d'enquête et a largement participé à l'élaboration des pistes d'analyse. Les auteurs remer- cient également Daniel Céfaï, Philippe Corcuff, Viviane Isambert-Jamati, Claire Joigneaux, Gabriel Nordacchione et Marie Salaün pour leurs lectures des versions antérieures de ce texte. 
lités, de niveau des élèves ou de moyens disponibles) et sur le lycée (lieu privilégié des stratégies familiales notamment en matière d'orientation des jeunes). Cependant les préoccupations commencent aujourd'hui à se déplacer au point que des mouvements protestataires significatifs concernent de plus en plus, quoique de façon moins exacerbée, l'école élémentaire (Dubet, 1997 ; Les dossiers d'Éducation et formations, 1998). Ces mobilisations de parents sont fréquemment soupçonnées de défendre des intérêts égoïstes ou des avantages acquis. Or non seulement ce soupçon ne permet pas de comprendre les ressorts de ce type d'action collective, mais il occulte les revendications de justice et les éventuelles formes de généralisation qui s'y manifestent.

La perspective initiée par Luc Boltanski et Laurent Thévenot (1991), et prolongée dans le domaine de l'école par Jean-Louis Derouet (1992), a mis l'accent sur la dynamique de la critique qui fonde le régime de la justification. Ce régime éclaire les conditions d'expression publique d'un sens ordinaire du juste et de l'injuste, à travers un processus de «montée en généralité », visant un bien commun. L'originalité de l'approche tient à l'identification d'une pluralité de sens ordinaire du juste et, par conséquent, de principes de justification susceptibles de se confronter dans un même univers. Des travaux empiriques en ont montré le caractère heuristique (Boltanski et Thévenot, 1989 ; Lafaye, 1990). Le processus de montée en généralité constitue cependant une condition d'accomplissement du régime de la justification qui mérite une interrogation renouvelée. Qu'en est-il lorsque la plainte ou la revendication sont indissociables d'un ancrage dans la proximité ? Ce dernier s'efface-t-il progressivement dans le travail de généralisation nécessaire à ce que la revendication soit acceptable dans l'espace public ou, à l'inverse, parvient-il à se maintenir et sous quelle forme? Les revendications centrées sur le maintien d'écoles élémentaires au sein du dispositif de zone d'éducation prioritaire (ZEP), parce qu'elles témoignent d'un ancrage dans un espace de proximité tout en cristallisant des revendications de justice, constituent un terrain privilégié pour tenter d'élucider le paradoxe des mobilisations de proximité.

Depuis le début des années quatre-vingt, en effet, existe en France un dispositif qui permet l'attribution de davantage de moyens aux écoles situées dans un quartier défavorisé, dit pour cette raison «zone d'éducation prioritaire » ou ZEP. Le nombre d'écoles ainsi mieux dotées a été limité, ce qui conduit les autorités de l'Éducation nationale à procéder à des ajustements, en fonction des évolutions repérées : de nouvelles écoles entrent dans le dispositif tandis que d'autres en sont retirées. Notre recherche s'est intéressée à un groupe scolaire situé dans un quartier de l'Est parisien, constitué respectivement de deux écoles maternelles de 5 et 10 classes et de deux écoles élémentaires de 8 et 13 classes (1). Les élèves concernés sont issus de populations socialement diverses, comme peut l'être lui-même ce quartier, habité à la fois par les milieux populaires (souvent d'origine étrangère) et par les classes moyennes (essentiellement intellectuelles). Le classement en ZEP a été

(1) Groupe désigné sous le simple nom de «groupe scolaire » dans la suite de l'article. 
obtenu pour les maternelles à la fin des années quatre-vingt, tandis que les écoles élémentaires n'ont posé leur candidature, sous la forme de vœux réitérés des conseils d'école, que plusieurs années plus tard pour obtenir finalement de l'académie et de la Ville de Paris un statut d'assimilé se traduisant par des moyens pédagogiques identiques à ceux des ZEP. Or, durant l'automne 1998, alors qu'une importante opération de révision des classements est en cours, il est fortement question que le groupe scolaire soit ramené au lot commun à la rentrée suivante. Cet article est centré sur la contestation que ce projet a déclenchée chez les parents.

À partir d'une enquête empirique (voir encadré) portant sur ce mouvement clairement délimité, nous examinons les modes d'engagement et de mobilisation des parents autour de l'école primaire, en prêtant une attention particulière aux relations établies à cette occasion avec l'ensemble des autres acteurs parties prenantes. L'analyse d'une intense mobilisation autour d'un groupe scolaire spécifique vise à mieux cerner les formes d'action collective qui prennent appui sur des ancrages locaux et des engagements de proximité. En effet, les transformations récentes de l'action publique et le mouvement de relocalisation qui affecte, depuis près de vingt ans, les politiques publiques s'accompagnent de l'émergence de nouvelles figures d'action collective articulées sur des territoires restreints et manifestant de la méfiance vis-à-vis des appareils institués et des formes d'action classiques (Chauvière et Godbout, 1992 ; Warin, 1997 ; Jeannot, 1998 ; Ion, 1999 ; Thévenot, 1999). Ces modes d'action, particulièrement visibles dans des domaines comme l'environnement ou l'aménagement urbain (Lolive, 1999; Céfaï et Lafaye, 2001), concernent également l'école (Dutercq, 2000) quoique sous des formes spécifiques que cet article se propose de cerner.

L'enquête s'est déroulée entre novembre 1999 et décembre 2000, soit plus d'une année après la mobilisation concernée. D'une part, a été réalisée une série d'entretiens semi-directifs auprès des principaux acteurs engagés dans cette mobilisation (membres des deux associations de parents d'élèves présentes sur le groupe scolaire, directeurs d'écoles et enseignants, inspecteur de l'Éducation nationale) complétée par des entretiens auprès de deux autres inspecteurs de l'Éducation nationale confrontés à des mobilisations similaires au sein de leur circonscription et par plusieurs discussions informelles avec des représentants de l'instance départementale de la FCPE. D'autre part, ont été collectées les traces documentaires produites à l'occasion de cette mobilisation (tracts, comptes rendus de réunions, bulletins des associations de parents d'élèves, articles de presse). À la différence de l'observation directe qui suit le déroulement d'une mobilisation dans son élaboration même et ses éventuels tâtonnements, les récits recueillis a posteriori tendent davantage à se présenter comme des intrigues narratives qui intègrent, dans une histoire cohérente, des événements multiples et dispersés (Ricoeur, 1983). Ce qui pourrait apparaître comme un biais méthodologique présente cependant l'intérêt de mettre d'emblée l'accent sur les éléments saillants, ceux dont les acteurs ont gardé la mémoire parce qu'ils ont structuré la mobilisation étudiée mais aussi parce qu'ils participent à la configuration du présent et sont, par conséquent, susceptibles de réemplois dans de nouveaux contextes (Lepetit, 1995 ; Corcuff et Sanier, 2000). Les récits ainsi recueillis, parce qu'ils sont, en partie, épurés des incertitudes liées à l'action, se prêtent tout particulièrement à l'identification d'une grammaire de l'action revendicative (Trom, 2001) comme de la justice scolaire. 


\section{L'investissement local et la proximité comme lieux pertinents de l'action}

Deux associations de parents d'élèves sont directement impliquées dans la controverse étudiée : un conseil local de la Fédération nationale des conseils de parents d'élèves (FCPE) et l'Amicale, une association construite sur le modèle du regroupement de proximité qui n'est affiliée à aucune fédération. L'Amicale comme le conseil local de la FCPE rassemblent leurs adhérents au niveau du groupe scolaire. Les deux associations en présence affichent plus de similarités que de différences : profils semblables des représentants élus et des adhérents (recrutés majoritairement parmi les couches sociales moyennes intellectuelles), valeurs identiques (laïcité, gratuité, solidarité), discours et façons de concevoir l'engagement qui se font écho. D'ailleurs, il n'est pas rare que des membres de l'Amicale aient été adhérents de la FCPE, dans une autre école, ou rejoignent celle-ci lorsque leurs enfants entrent dans le secondaire.

L'orientation des deux associations traduit, en effet, une conception de l'engagement, et plus généralement de l'action politique, empreinte de défiance à l'égard des grands appareils organisés, directement articulés sur le débat politique national (partis, syndicats, grandes fédérations associatives). La FCPE fait ainsi l'objet d'un ensemble de critiques qui émane tant des animateurs de l'Amicale que de ceux de son conseil local. Contrairement à la dichotomie couramment observée dans des situations similaires, la tension entre un bien commun local (restriction de l'horizon du débat scolaire aux murs de l'école fréquentée par ses propres enfants) et un bien commun attentif à des exigences plus vastes ne constitue pas une ligne de partage entre les deux associations de parents d'élèves en présence. Les propos tenus par leurs animateurs respectifs attestent de l'importance d'un investissement ancré dans une expérience localisée. Est-ce à dire que cet investissement se limite aux murs de l'école ou du groupe scolaire ? Nous voudrions montrer que cette mobilisation de parents témoigne d'un accès revendiqué à la généralisation à partir d'une expérience localisée et aspire à interroger le bien-fondé d'une politique publique nationale à partir de sa mise en œuvre sur un territoire restreint.

\section{Une fédération nationale trop peu à l'écoute de ses conseils locaux?}

Un des animateurs de l'Amicale, bien qu'il se dise politiquement proche de la FCPE - sa compagne en est adhérente au collège - critique l'insuffisante prise en compte des conseils locaux par les instances départementales et nationales de la FCPE : «J'avais des échos qui m'ont fait réfléchir sur le fonctionnement de la FCPE, sur son fonctionnement interne en tant qu'association. [...] Ils semblaient assez bureaucratiques, ne prenant pas en compte ce qui se passe dans les conseils locaux. » Les raisons énoncées suggèrent que 
la vie démocratique est étrangère aux grandes structures et ne se réalise pleinement qu'au sein d'une petite structure agissant sur un territoire circonscrit (2). La critique du déficit démocratique est présentée comme étant au fondement de la création de l'Amicale, née d'une scission du conseil local FCPE une dizaine d'années auparavant. Deux motifs sont pointés, qui se renforcent l'un l'autre : le rejet de l'instrumentalisation politique (la FCPE comme élément du réseau des partis politiques de gauche et, plus spécifiquement, du Parti socialiste) et la difficulté de déploiement des actions locales perçues comme fréquemment bridées par les instances fédérales.

La critique de l'insuffisante prise en compte des conseils locaux est également présente, quoique sous une forme sensiblement différente, dans les entretiens réalisés avec des membres du conseil local de la FCPE. En effet, c'est notamment au moment de la mobilisation en faveur du maintien en ZEP qu'ils font l'expérience plus ou moins aiguë d'un désajustement entre le combat qu'ils engagent au niveau local et la position des instances supérieures de la FCPE. Si certains membres du conseil local FCPE font d'emblée état d'une "grosse divergence d'appréciation» quant au bien-fondé du redécoupage de la carte des ZEP parisiennes, d'autres sont davantage sensibles au déficit de relations entre les conseils locaux et les instances départementales. « La FCPE au niveau de Paris a sans doute négocié les choses avec l'Éducation nationale, le seul problème, c'est que ça n'est strictement pas redescendu [...] C'est le type même d'un fonctionnement non démocratique : on va négocier et les gens qui sont les mandants n'étaient même pas au courant qu'ils avaient négocié quoi que ce soit. »

La critique porte explicitement sur le fonctionnement de la structure FCPE et nullement sur ce qui a été négocié puisque cet adhérent de base manifeste, par ailleurs, son accord avec les positions départementale et fédérale et fait état de ses réserves personnelles sur l'action engagée au moment de son impulsion. Ce qui est dénoncé réside dans le fait que les instances supérieures négocient sans consulter préalablement leur base, ne rendent pas compte du résultat de ces négociations et, de surcroît, lorsque la mobilisation est lancée, omettent d'expliciter le bien-fondé de leur position, rendant tout débat contradictoire impossible.

Engagés dans l'action en faveur du maintien en ZEP de leurs écoles, les membres du conseil local FCPE font alors l'expérience directe de l'absence de soutien des instances départementale et fédérale, évoqué dans le récit fondateur de la création de l'Amicale. Cette absence de soutien se traduit de façon concrète par un refus d'appui logistique, de relais d'informations et de coordination, éléments qui font habituellement la supériorité des grandes

(2) À l'origine, les fédérations de parents d'élèves se sont constituées à partir de la mise en réseau d'associations de parents créées dans tel ou tel établissement : c'est le cas de la PEEP (enseignement public) ou de l'UNAPEL (enseignement privé catholique). La FCPE, d'obédience de gauche, a une origine en partie différente puisqu'elle est née après la Seconde Guerre mondiale de la volonté de grands mouvements nationaux (SNI, Ligue de l'enseignement), tout en se construisant également sur la base d'une fédération d'associations locales. 
organisations fédérales lorsqu'il s'agit de mener une action collective. Ce déficit et le désaccord qui le motive sont découverts dans le déroulement même de l'action et éprouvés au fur et à mesure des attentes déçues. La conséquence en est une dramatisation des relations entre les conseils locaux et l'instance départementale : "On a essayé de faire que la FCPE joue un peu un rôle de coordination ; on s'est aperçu qu'elle freinait par tous les moyens. » La mobilisation se constitue alors en dehors de l'aide des grandes fédérations nationales de parents d'élèves et les conseils locaux de la FCPE engagés dans cette controverse sont conduits à former une coordination des écoles concernées avec les groupements locaux de parents.

Cette expérience de l'isolement, vécue par les adhérents du conseil local de la FCPE, témoigne des difficultés à parler d'une même voix qui caractérisent les fédérations de parents d'élèves, tiraillés entre les mots d'ordre très généraux de leur «centrale » et les attentes plus pragmatiques de leurs adhérents (Dutercq, 2001). Le responsable du conseil local évoque un fonctionnement clivé : «La plupart des parents d'élèves sont investis sur leur école et ne revendiquent pas, ne cherchent pas à s'investir dans la constitution d'une fédération de parents d'élèves. Comme souvent dans ce genre de cas, ceux qui se retrouvent en situation de [s'investir dans une fédération] sont plutôt des gens qui ont un projet politique. »C'est ce que confirme à sa manière un autre membre du conseil local : "Comment les représentants sont élus, comment ils y arrivent... Je n'en sais absolument rien. J'en ai aucune idée et je m'en fous à la limite... Je n'ai pas le temps : on est parent à plein temps, mais on n'est pas représentant de parents d'élèves à temps plein. » La plupart des adhérents de la FCPE tendent, à l'instar de cet interviewé, à se désinvestir du fonctionnement départemental et fédéral, au profit d'un investissement exclusif au sein du conseil local, ce qui rapproche leur engagement de celui des adhérents des groupements locaux de parents. L'orientation locale de l'engagement des parents d'élèves signifie-t-elle, pour autant, que leur champ d'intervention est borné aux murs de l'école?

\section{Une clôture à géométrie variable du champ pertinent d'intervention}

Les propos des membres de l'Amicale interrogés attestent de l'existence de conceptions différentes du champ d'intervention pertinent. D'une part, l'Amicale s'autodésigne explicitement comme une association locale dont le champ d'intervention tend à se clôturer pour chaque adhérent sur l'école, voire le groupe scolaire fréquenté par son enfant. L'école est conçue comme le lieu à la fois pertinent et souhaitable de ce qui peut être décidé et mis en œuvre à propos de la scolarité de son enfant : "L'idée c'est que c'est des parents qui ont envie de s'investir dans la scolarité, les décisions se prennent là et pas ailleurs et que les choses se font en fonction de la réalité de cette école, qu'on est pas forcément obligé de se positionner systématiquement sur tout et tout le temps quand on ne se sent pas concerné. » D'autre part, à cette conception d'un groupement de parents d'élèves préoccupés des seules ques- 
tions scolaires se superpose une autre conception orientée sur la vie de quartier, conception qui motive l'adhésion à l'Amicale de ce parent d'élèves : «C'était une association locale qui était d'abord une association de quartier qui avait aussi une action sur la vie du quartier, ça m'intéressait [...] Les associations de parents d'élèves, ici en tout cas, je ne sais pas ailleurs, c'est un bon moyen à la fois d'être au courant de ce qui se passe dans le quartier et puis d'avoir l'occasion de s'investir, de participer à des choses. » La présidente de l'Amicale développe une conception identique : «J'ai choisi d'entrer à l'Amicale parce que c'est une association de quartier ; moi, ce qui m'intéressait, c'était de connaître des gens du quartier, de défendre des idées dans mon quartier sachant qu'il y a des limites à être dans une association de quartier et pas dans une association nationale. » Élargi au quartier, le champ pertinent d'intervention de l'Amicale peut s'ouvrir à d'autres questions que seulement scolaires. Militer à l'Amicale, c'est participer à un engagement de proximité qui trouve divers lieux d'exercice (association de quartier, conseil de quartier, etc.) et resituer un investissement spécifique dans un engagement élargi à d'autres préoccupations que strictement scolaires.

Les adhérents du conseil local de la FCPE, s'ils y ont adhéré parce qu'ils se sentent souvent proches de ses grandes orientations politiques, notamment sur la laïcité, et s'ils ont plus souvent qu'au sein de l'Amicale un passé ou un présent militant (3), tendent également à articuler leur participation à une association de parents à un engagement par rapport à la vie du quartier et non pas uniquement à l'école. Tous mentionnent leur investissement dans des associations de quartier ou dans le conseil de quartier. Le conseil local de la FCPE a d'ailleurs revendiqué très tôt son engagement dans cette instance participative, instituée dans l'arrondissement dès 1995, au prétexte que l'école est directement concernée par les mutations de son environnement. C'est ainsi que l'évolution urbaine du quartier est une préoccupation constante pour les représentants du conseil local de la FCPE : «Je suis préoccupée quand même par les problèmes d'urbanisme du quartier [...] C'est-à-dire qu'on construit à tour de bras dans le quartier sans construire d'équipements, sans tenir compte des problèmes sociaux qui sont, pour moi, très liés à l'urbanisme. » Ils ont attiré, à plusieurs reprises, l'attention de la mairie et de l'inspecteur de l'Éducation nationale sur les projets d'urbanisation du quartier et leurs répercussions sur les effectifs scolaires.

Les militants des deux associations expriment une rhétorique de l'engagement présentant de grandes similitudes. Cet investissement sur le quartier, commun au conseil local de la FCPE et à l'Amicale, suggère une autre manière de généraliser. À côté de la verticalité, qui passe par une spécialisation des domaines d'intervention (les fédérations de parents ne s'occupent que de l'école) mais les traite à l'aide de réseaux longs, l'horizontalité, incarnée par l'investissement élargi de l'école au quartier, vise à étendre, à diversifier les domaines d'intervention et à construire plutôt des réseaux courts mais denses. Cette seconde manière de généraliser implique la déspécialisation. Elle n'est pas le propre des seuls regroupements locaux de parents d'élèves

(3) Le responsable du conseil local FCPE se définit d'emblée comme syndicaliste, permanent syndical à SUD, un autre est, depuis 25 ans, adhérent de la CFDT. 
puisque, sur le terrain étudié, elle concerne tout autant les adhérents du conseil local de la FCPE, impuissants à rendre opérants leurs réseaux longs. Cependant, dans la mobilisation étudiée, émerge également une troisième manière de généraliser qui consiste à faire d'un cas un exemple (Desrosières, 1989, 1993a, 1993b ; Céfaï et Lafaye, 2001). L'ancrage dans la situation localisée permet de poser à la politique engagée, en prenant appui sur des éléments concrets, un ensemble de questions générales. Mais avant cela, il convient d'examiner sur quel terreau émerge le sentiment d'injustice qui va constituer l'aiguillon propice au développement de la mobilisation.

\section{L'émergence d'un sentiment d'injustice}

La mobilisation étudiée se constitue autour d'un sentiment d'injustice. D'une part, celui-ci émerge en réaction à une mesure académique. La décision de retirer le statut ZEP à certaines écoles est perçue comme autoritaire et imposée d'en haut, incarnation d'une conception substantialiste de l'action publique (Padioleau, 1993) selon laquelle l'administration est seule dépositaire de l'intérêt général. D'autre part, le sentiment d'injustice embraye sur des circonstances locales, en l'occurrence une méprise sur l'objet d'une réunion. C'est sur ce terreau que prend corps la mobilisation dont nous dégagerons une partie du travail argumentatif produit par les acteurs pour faire valoir l'injustice qui leur est faite. Le cadre d'injustice développé dans et par ce travail argumentatif favorise la mobilisation collective et contribue à mouvoir autour d'une cause les personnes qui vont se l'approprier. Au fur et à mesure que la mobilisation gagne en ampleur, il devient plus identifiable et plus solide car de mieux en mieux argumenté.

\section{Une conception substantialiste du service public éducatif}

La relance de la politique des ZEP s'appuyait, aux dires de l'inspecteur de l'Éducation nationale interrogé, sur une évaluation qui impliquait un reclassement général opéré à moyens constants des écoles sortant du dispositif pour permettre à d'autres d'y entrer. L'inspecteur de l'Éducation nationale se situe, d'emblée, dans une logique d'intérêt général dont les éléments ont été annoncés publiquement dans le cadre du Pacte de relance des ZEP, même si le bien-fondé de cette logique échappe à ceux qu'elle lèse individuellement, soit qu'ils l'admettent mais à condition qu'elle ne s'applique pas à eux, soit qu'ils refusent l'idée même d'un redéploiement qui ne s'accompagnerait pas simultanément d'un accroissement des moyens. L'analyse du point de vue de l'inspecteur témoigne d'une conception du juste fondée sur un compromis civique-industriel, selon lequel l'administration est l'exécutif technique d'une volonté politique qui la dépasse (4). Dans cette conception substantialiste,

(4) En ce sens, la terminologie wébérienne d'autorité à caractère rationnel-légal désigne on ne peut mieux ce compromis entre les deux principes de justification sur lesquels s'est fondé l'État moderne. 
l'administration incarne l'intérêt général en raison de sa vision globale des situations et des problèmes. Elle n'a pas, selon l'inspecteur, d'intérêt propre à défendre : elle recueille des éléments, elle mesure, elle compare, elle essaie d'établir des critères de référence, au nom desquels elle répartit : « Alors moi, effectivement, j'aurais tendance à penser que quand je fais des propositions à l'inspecteur d'Académie, c'est à partir d'une vision globale. » L'administration («académique » voire «centrale », l'inspecteur ne fait pas la différence) travaille donc dans le sens de l'efficacité, mais une efficacité réfléchie, critériée, en un mot, juste. Gestion rationnelle (apanage de l'administration) et recherche de l'intérêt commun s'y confondent, même s'il est difficile de le faire admettre : «Ça me semble exemplaire de la difficulté pour l'administration de l'Éducation nationale et peut-être pour l'administration de l'État dans son ensemble à faire comprendre qu'un certain nombre de décisions sont prises dans l'intérêt collectif. » L'administration pêche parfois par défaut de communication, mais ses projets et décisions sont avant tout animés par une rationalité qui, si elle peut paraître purement gestionnaire aux yeux de ses détracteurs, n'est pour l'inspecteur que l'expression de la recherche de l'intérêt collectif. Du reste cet inspecteur se présente, tout au long de l'entretien, comme le garant, sur sa circonscription, d'une répartition juste des moyens alloués.

Dans cette perspective, les mobilisations locales sont alors dénoncées comme la défense d'intérêts de groupe contre l'intérêt général auquel elles sont aveugles car elles n'ont pas cette vision globale que revendique l'administration. Les mouvements de parents sont décrits par l'inspecteur comme centrés sur la défense de ceux qui les animent, principalement des représentants des classes moyennes : «Le discours des parents du groupe scolaire est certainement plus près au fond du sentiment des couches moyennes qui veulent bien rester dans un certain nombre de quartiers mais pas sacrifier leurs enfants puisqu'ils présentaient cela comme sacrifier leurs enfants. » La conception substantialiste de l'action publique dénie aux usagers comme aux personnels de base la capacité d'émettre un point de vue autre que celui de la défense de leur intérêt particulier. Ce faisant, elle est dans l'incapacité d'envisager la justification des mesures qu'elle prend et, a fortiori, leur négociation à l'instar d'autres administrations (Fourniau, 1996). Cette posture de l'administration suscite des réactions d'autant plus vives qu'elle s'inscrit dans le contexte d'une méprise concernant l'objet de la réunion où la mesure est annoncée.

\section{Un catalyseur d'injustice}

En effet, les éléments exposés constituent une conjoncture mais encore faut-il, pour cristalliser rancœurs et déceptions, une situation, un microévénement qui servira d'élément déclencheur: la manière dont parents et enseignants sont informés que leurs écoles perdent le statut ZEP va offrir le stimulus nécessaire, apte à mobiliser le plus grand nombre. Tous évoquent le 
choc qu'a constitué la réunion organisée à l'initiative de l'inspection académique, au cours de laquelle leur est annoncée cette nouvelle. Ce choc résulte d'une méprise quant à l'objet de la réunion, ce qu'Erving Goffman qualifie de « rupture de cadre » (Goffman, 1991, pp. 338-369). Alors que les personnes invitées (directeurs d'école, enseignants, représentants de parents) croient assister à une réunion générale présentant les nouvelles orientations de la politique des ZEP, elles découvrent, en situation, tout à la fois que les écoles qu'elles représentent perdent le statut de ZEP et que, de surcroît, n'ont été conviés à cette réunion que les représentants des écoles déclassées.

Les propos tenus par l'une des directrices d'écoles maternelles retracent, avec beaucoup de détails, le contexte de cette rupture de cadre : « Nous, on avait déjà beaucoup réfléchi l'année d'avant puisqu'il y avait eu le fameux forum de relance des ZEP par Mme Ségolène Royal. J'y avais été un mercredi toute la journée parce que j'étais vraiment intéressée par cette relance... Septembre 98, ça je me souviens : on nous annonce une réunion avec M. B qui est l'inspecteur de l'Éducation nationale chargé des ZEP, donc il réunissait le 5 octobre un certain nombre d'écoles pour faire le point sur les ZEP et c'est vrai que là j'avais mobilisé les troupes... Nous arrivons toutes la bouche en cœur et en fait M. B. nous annonce que certaines écoles en fonction des critères établis et nous n'avions évidemment pas été du tout consultés, qu'on sortait de la ZEP, alors je dois dire que là quand même ça a été comme un coup de massue. Je me souviens seulement qu'au cours de la réunion j'ai traité M. B. d'énarque mais c'est tout ce dont je me souviens de cette réunion parce que vraiment on s'était toutes retrouvées avec l'idée de retravailler, de comment travailler en ZEP, et on apprend qu'on en sort. » Cette rupture de cadre est confirmée par les propos des parents : «Je crois qu'au début, c'est quand même parti sur un malentendu [...] Donc on s'est trouvé dans une réunion où on ne savait pas trop de quoi on allait nous parler, on s'attendait surtout pas qu' on nous parle de ça, qu' on allait nous enlever notre statut de ZEP. » L'inspecteur en charge de la circonscription admet pour sa part une petite maladresse tout en affirmant que les objectifs de la réunion, contrairement à ce qu'affirment parents et enseignants, étaient clairs : « Il y a eu un malentendu, nous il nous semblait que l'explication de la nouvelle politique des ZEP avait été faite puisqu'il y avait déjà eu la rencontre de Rouen, qu'il y avait un certain nombre de documents qui avaient circulé, bon, peut-être qu'on n'en fait jamais suffisamment hein !... Peut-être qu'il y a eu une petite maladresse. »

La réaction ulcérée dont témoignent les représentants des écoles présents à la réunion (qu'ils soient enseignants ou parents) met en évidence la modification des attentes à l'égard de l'administration d'État et de ses représentants : les personnels en contact direct avec les usagers (ici les enseignants responsables des écoles) comme les usagers eux-mêmes refusent la toute-puissance d'une administration perçue comme arrogante et immatérielle, qui prend des décisions sans avoir besoin de les justifier. Le fonctionnement par voie de circulaires, de décisions non négociables, qui constituait l'essentiel des rapports entre administration et assujettis, est de plus en plus contesté au 
profit d'un fonctionnement fondé sur la négociation et la contractualisation, ce qui donne une responsabilité déterminante aux hiérarchies intermédiaires de l'État. L'élément déclencheur, c'est peut-être moins la décision elle-même que la manière dont l'administration traite ceux qui y sont assujettis.

\section{Travail argumentatif et caractérisation de l'injustice}

La décision de l'administration de l'Éducation nationale de retirer le statut ZEP aux écoles maternelles du groupe scolaire suscite un sentiment d'injustice exacerbé chez la plupart des parents et des enseignants. Ce sentiment est alimenté par plusieurs éléments qui, progressivement, sont travaillés, argumentés, étayés et portés dans un espace public de débat, la mobilisation et les discussions qui l'ont accompagnée faisant progressivement émerger un argumentaire commun. C'est ce travail argumentatif, réalisé par les acteurs pour caractériser l'injustice subie, que nous essaierons de décomposer analytiquement en en pointant les nuances.

En premier lieu, la caractérisation de l'injustice porte sur la relation entre la perte du statut ZEP et la situation socioprofessionnelle des familles scolarisées dans le groupe scolaire. Le déclassement est ainsi jugé particulièrement injuste par des représentants de parents qui perçoivent l'environnement social des écoles comme défavorisé : «Il y avait une sorte de notion de se dire : bon, pourquoi nous, on n'est quand même pas dans les quartiers les plus privilégiés. » Les équipes pédagogiques évoquent même une dégradation de cet environnement social : "Nous on trouvait ici que c'était injuste, qu'on nous sorte de ZEP alors qu' on trouve au contraire que le quartier se dégrade, c'està-dire qu'il y a quand même une fuite des familles qui n'ont pas trop, trop de difficultés et il y a de plus en plus d'arrivées de familles en difficulté. »

Les écoles concernées ont été classées ZEP en raison de leur situation : elles scolarisent les enfants d'une importante cité populaire. Pour les enseignants et les parents, les difficultés sociales qui ont justifié l'octroi de ressources supplémentaires se sont plutôt aggravées en raison de l'installation de nouvelles populations dont les caractéristiques (par exemple l'ignorance de la langue française, sur laquelle insiste particulièrement un membre de l'Amicale) nécessitent une prise en charge de plus en plus importante (5). De surcroît, pour des raisons d'absence de collecte centralisée et informatisée de la nomenclature professionnelle usuelle, l'indicateur utilisé par l'administration de l'Éducation nationale pour repérer la situation socioprofessionnelle des familles correspond à la répartition entre les différents tarifs de cantine, indicateur controversé par les directeurs, les enseignants et les parents qui en

(5) L'augmentation constante du nombre de familles en difficulté est également mentionnée par l'assistante sociale du groupe scolaire, année après année, comme en témoignent les comptes rendus de conseils d'écoles. L'objec- tivité de cette donnée doit être relativisée dans la mesure où le constat effectué peut simplement refléter une montée en charge, au fil des ans, de l'activité de l'assistante sociale. 
pointent les faiblesses, les familles les plus défavorisées envoyant rarement leurs enfants manger à la cantine.

Deux variantes à cette première formalisation de l'injustice subie peuvent être repérées. Sans contredire la formalisation précédente, elles intègrent le fait que le recrutement social des écoles n'est pas homogène puisque y sont également scolarisés des enfants issus des classes moyennes. Toutes deux mettent l'accent sur le fait qu'il est injuste de réduire les ressources à la disposition des écoles dont une partie seulement des élèves est considérée comme défavorisée, sous le prétexte qu'il existe des écoles à la population exclusivement défavorisée. Les élus communistes locaux, qui ont clairement soutenu le mouvement, en présentent dans leurs tracts un premier énoncé : «Ce que propose l'administration, c'est de déshabiller Pierre pour habiller Paul. » (6). Une des directrices d'école maternelle l'explicite en des termes qui mettent l'accent sur les bénéfices du statut ZEP pour les enfants les plus démunis du quartier: «On avait ce mélange de populations qui faisait qu'on allait redonner de l'argent à d'autres écoles qui apparemment ou effectivement ont plus de difficultés que nous. Mais nous on prouvait que le fait qu'on soit en ZEP, justement, avec une population mélangée, le fait qu'on soit limité par exemple au niveau des effectifs, ça nous permet malgré tout d'accueillir dans de bonnes conditions des enfants en difficulté et on trouvait pas normal qu'on nous ôte une aide qui nous permet justement de maintenir cette sorte d'équilibre. » Le sentiment d'injustice provient ici, comme précédemment, du fait que la mesure prise fait peser une menace sur la scolarité des enfants les plus défavorisés du groupe scolaire.

La seconde variante repérée place l'injustice sur le fait que ce sont des quartiers et des groupes scolaires socialement mélangés mais connaissant néanmoins des difficultés qui sont sacrifiés au profit de plus défavorisés qu'eux et non les écoles des quartiers les plus favorisés : «Je crois aussi que les gens se disaient pourquoi prendre à des quartiers populaires et pourquoi pas prendre au XVI

En second lieu, la caractérisation de l'injustice porte sur la relation entre le statut ZEP, le milieu social des familles et le faible taux de retard scolaire des élèves des écoles menacées de déclassement. Parmi les critères retenus pour proposer le maintien ou la sortie de ZEP, l'interprétation, faite par l'administration de l'Éducation nationale, du faible taux de retard scolaire des élèves du groupe scolaire suscite un puissant sentiment d'injustice chez les parents et les enseignants. Pour les services académiques, un taux de retard inférieur à la moyenne des écoles situées en ZEP justifie le déclassement parce qu'il conforte l'hypothèse qu'une partie des élèves est issue de milieux sociaux favorisés. Les taux de retard et de réussite scolaires sont conçus, dans cette perspective, à l'instar de nombreuses analyses de sociologie critique, comme la traduction mécanique du déterminisme du milieu social. Cette analyse provoque révolte et colère chez les parents et les enseignants dans la mesure

(6) Une représentante du conseil local FCPE interrogée a également recours à cette expression imagée. 
où le déterminisme qui l'inspire nie tout à la fois le bénéfice des moyens supplémentaires octroyés dans le cadre du statut ZEP et le travail des enseignants pour surmonter les handicaps liés au milieu social. Les parents, tant de l'Amicale que de la FCPE, font ainsi valoir que ce sont peut-être les moyens supplémentaires affectés dans le cadre de la politique d'éducation prioritaire qui permettent à certaines familles du quartier de faire face aux difficultés sociales, culturelles et linguistiques et aux enfants qui en sont issus de réussir leur scolarité. «On s'est rendu compte, au fil des années, que ces enfants qui avaient du mal à parler français, qui avaient pas mal de difficultés, petit à petit et grâce justement aux moyens supplémentaires de la ZEP, arrivaient quand même à un niveau assez convenable. » Le président du conseil local FCPE résume de façon imagée l'injustice ressentie en évoquant une «prime au ratage » : «On avait constaté une amélioration des résultats pédagogiques et une amélioration du nombre d'enfants qui aboutissaient à une scolarité normale. On avait l'impression que c'était une prime au ratage. Pour être en ZEP, il fallait continuer à rater. »

À ce deuxième élément s'en ajoute un autre, connexe, qui met l'accent sur la pénalisation de l'investissement des enseignants : il est injuste et décourageant de retirer les moyens accordés à ceux qui ont montré leur engagement, et en partie réussi, grâce à un surcroît de travail. Cet argument met en question la logique d'attribution de ressources supplémentaires, dont une prime aux enseignants des écoles en ZEP. Comme l'attribution de moyens pédagogiques supplémentaires, la prime n'a plus de raison d'être, une fois l'objectif atteint. Or les enseignants des écoles visées assimilent cette prime comme partie intégrante des moyens accordés aux ZEP et considèrent sa suppression comme une sanction: «C'est cette méconnaissance du travail des gens qui nous a choqués [...] Dans l'administration, on oublie l'humain parce qu'en fait il y a des circulaires avec des programmes mais ce qu'on ne voit pas c'est le travail qui est fait de façon souterraine pour pouvoir mettre en application tout ça. En tout cas c'est ce que ressentaient les enseignants, une sorte de négation de leur travail, de leur engagement. »

L'articulation entre ces différents arguments permet de dessiner un cadre d'injustice propice au développement d'une mobilisation collective. S'y trouvent conjugués des éléments qui concernent l'ensemble des acteurs locaux, fondés sur un sens commun du juste et désignant notamment des victimes (les enfants les plus défavorisés du groupe scolaire) et un persécuteur (l'administration de l'Éducation nationale). Obéissant au système de contraintes formelles de la dénonciation (Boltanski, 1990, pp. 266-279), le travail argumentatif opère un premier découplage entre les dénonciateurs (les représentants des parents mais aussi les enseignants) et les victimes (les enfants défavorisés du quartier). À ce stade, l'injustice subie est constituée dans un va et vient continu entre des repères de proximité (une connaissance de l'école et du quartier fondée sur l'usage et la fréquentation ; des moyens pédagogiques face auxquels on met en regard des réussites d'enfants clairement identifiés ; une prime perçue comme la reconnaissance d'un engagement dans l'activité pédagogique) et des repères plus éloignés qui conduisent les acteurs à établir 
des rapprochements, à mettre en équivalence des écoles de quartiers différents. Ce sont là les prémices d'un processus de montée en généralité.

\section{Mobilisation autour de la ZEP et montée en généralité}

Pour être entendue, la mobilisation doit trouver les moyens d'accéder à une arène publique : elle doit faire la preuve de la légitimité de ses revendications et, pour cela, faire obstacle au soupçon de défendre des intérêts particuliers.

\section{Revendication égoüste et argumentation recevable}

La mobilisation autour du maintien en ZEP est d'emblée confrontée au soupçon d'une revendication égoïste, attachée à la défense d'avantages acquis. L'inspecteur en charge de la circonscription évoque deux éléments discordants par rapport à une tentative d'élaboration d'une cause au-dessus de tout soupçon. D'une part, les enseignants travaillant dans une école située en zone d'éducation prioritaire bénéficient d'une prime à laquelle ils ne pourraient plus prétendre dans le cas d'un déclassement; d'autre part, les parents rencontrés dans le cadre de la mobilisation lui ont semblé être des parents issus des classes moyennes, soucieux avant tout de conserver les avantages matériels liés au statut ZEP. Le soupçon d'être mû par son intérêt particulier participe de la dynamique de ce type de mobilisation. Il est intégré par les acteurs qui s'y trouvent engagés, lesquels sont alors contraints à rechercher et à développer des arguments dont la recevabilité n'est pas contestable (Trom, 1999).

On a vu précédemment que la menace pesant sur la prime enseignante avait pu servir d'appui à la constitution de l'injustice subie chez les équipes pédagogiques. Cependant, si la revendication du maintien de cette prime peine à trouver sur le moment une expression publiquement recevable, les entretiens réalisés a posteriori se prêtent davantage à sa défense : "Je ne vois pas quelle personne accepterait pour fournir le même travail de se voir diminuer son salaire, je trouve toujours un peu étonnant qu'on trouve anormal que les gens se battent aussi pour être payés correctement en fait. » La défense, dans le registre syndical, de sa légitimité, à l'instar des propos tenus par cette directrice d'école maternelle, n'a pas eu d'écho public lorsque la controverse a pris corps et s'est déroulée, ce qui ne signifie pas qu'elle n'aurait pas été recevable au sein d'autres mobilisations. L'autre directrice, tout en faisant valoir le bien-fondé de cette prime qui récompense un investissement, tient des propos qui attestent d'une prise en compte du soupçon pesant sur l'engagement des personnels éducatifs au sein de cette mobilisation: «Les premiers temps, beaucoup de personnes ont cru qu'on se battait pour ces 575 francs. En fait, très vite les gens ont compris que c'était pour garder une certaine qualité d'école et que par contre je l'ai redit souvent, je suis désolée, ça a quand 
même demandé de la part des enseignantes un investissement vraiment important et je ne vois pas pourquoi on irait cracher sur 575 francs. C'est péjoratif, vous comprenez ce que je veux dire, il n'y a que les gens bourrés de fric qui disent que le fric ne compte pas. » Le récit rétrospectif permet ici de faire tenir ensemble ce qui, au moment de la mobilisation, ne pouvait être rassemblé : d'un côté, le développement réussi d'une argumentation faisant échec au soupçon engendré par l'existence de cette prime, d'un autre côté, la justification du bien-fondé de cette même prime.

Le soupçon d'une mobilisation centrée sur la défense d'avantages acquis se porte également, on l'a vu, à l'encontre des parents, suspectés d'être attentifs aux conditions de scolarité de leurs seuls enfants. Ce soupçon est d'autant mieux pris en compte, dans le cas étudié, qu'il est partagé par quelques parents, au début de la mobilisation : «Je crois que c'était à l'origine une mobilisation très localo-locale : on touche à nos avantages [...] Moi au départ, j'étais assez réservé sur l'action parce qu'effectivement, il y des situations beaucoup plus désespérées. Je travaille en Seine-Saint-Denis, je crois que c'est clair... donner les mêmes moyens qu'à Stains ou à La Courneuve, je trouve ça un peu déplacé. » Émanant d'un représentant de la FCPE, qui manifeste ses doutes, dans les premières réunions rassemblant parents et enseignants, ce soupçon va servir d'aiguillon à la mobilisation, obligeant ce faisant les associations de parents à élaborer une argumentation qui vaille en toute généralité. Le président du conseil local de la FCPE, lorsqu'on l'interroge sur la façon dont la mobilisation a pris corps, fait explicitement état du rôle joué par cette critique dans la recherche d'arguments propres à la faire taire : « $\mathrm{Au}$ sein de la FCPE, il y a eu un clivage au départ avec un adhérent qui était très pour l'idée d'une discrimination positive qui devrait un jour disparaître parce qu'elle aurait porté ses fruits [...] On a réfléchi, cherché, travaillé avec les enseignants pour trouver tous les arguments, qu'on a portés en tant que tels auprès de l'administration, qui ne nous a pas du tout écoutés mais qu'on a portés en tant que tel. »Comment les parents et les enseignants qui revendiquent le maintien en ZEP le justifient-ils ? Avec quels arguments ou selon quels procédés peuvent-ils faire échec au soupçon d'égoïsme qui pèse sur la cause qu'ils défendent?

\section{La montée en généralité par la solidarité}

Parmi les nombreux éléments mis en avant dans le cadre de cette mobilisation, un argument récurrent a consisté à faire valoir que ceux qui se sont mobilisés ne l'ont pas fait pour eux-mêmes, pour leur confort d'enseignant ou celui de leurs propres enfants, mais bien pour les enfants des familles les plus démunies du quartier, lesquelles, précisément, ne se mobilisent pas. On peut distinguer chez les parents deux figures de cette montée en généralité par la solidarité.

La première figure, incarnée par une adhérente de l'Amicale, se clôture d'emblée sur l'espace de voisinage, pensé sur le modèle communautaire du 
village dans la ville : "C'était un mouvement très, très fort ! C'était la première fois qu'on a pu mobiliser comme ça. Même les gens qui, en fait, n'étaient pas du tout concernés, qui pouvaient suivre leur gamin ou qui avaient aussi des moyens financiers, étaient eux en premier mobilisés pour défendre ceux qui n'ont pas cette possibilité-là. Il y avait... Enfin, moi j'appelle cela un village notre secteur, parce que c'est vrai qu'il y a une grande solidarité. »Cette solidarité est, dans le même temps, marquée par l'incomplétude, non seulement en raison de l'absence de ceux pour lesquels on se bat, mais parce que le travail de mobilisation en leur direction est jugé insuffisant: «En fait moi, j'ai trouvé dommage, dans ce mouvement, que c'était pas forcément les parents qui avaient besoin que l'école soit en ZEP qui étaient là. J'ai trouvé ça dommage. »Cette conception de la solidarité exprimée par une adhérente de l'Amicale, exigeante sur le plan de l'élargissement tant de la mobilisation que du recrutement des associations de parents d'élèves, débouche sur le constat critique que l'association à laquelle elle a adhéré privilégie trop l'entre-soi : «Non, je ne pense pas que ce soit une volonté d'exclure ces gens-là. Je pense qu'il n'y a pas assez de travail de fond qui est fait... Moi, c'est ça qui me gêne, se retrouver entre gens de même niveau social, moi, ça me dérange ; j'aurais aimé que ce soit plus large.» Partant d'une conception communautaire de la solidarité, les propos tenus aboutissent cependant à une critique politique du fonctionnement des associations de parents d'élèves.

La seconde figure, moins développée, ouvre sur une vision où la solidarité est exprimée comme un ressort explicite de la mobilisation et articulée à des valeurs politiques de gauche. Voici par exemple ce que dit la présidente de l'Amicale : «Donc, je dirais qu'après le mouvement est parti, ce n'était pas tellement pour nos enfants qui n'ont pas spécialement de problèmes scolaires. C'était peut-être plus pour les autres, c'est là aussi où ça a rejoint le côté, je sais pas... des mouvements de gauche. » Ces propos témoignent d'une trajectoire inverse du cas précédent : la solidarité politique évoquée, à visée civique universaliste, tend paradoxalement à se clôturer sur un périmètre restreint, « les autres » désignant clairement les enfants défavorisés du groupe scolaire. Dès lors, la montée en généralité soutenue par les deux figures de la solidarité identifiées, si elle permet de faire échec à l'accusation d'égoïsme, présente, dans ce cas, une forme d'inachèvement liée à sa restriction à un territoire géographiquement délimité.

\section{Quelques questions générales posées par une mobilisation locale}

Les entretiens auprès des parents, qui restituent les temps forts du travail argumentatif réalisé pour faire valoir leur revendication, font état d'une ambition sinon de repenser l'ensemble de la politique des ZEP, du moins de pointer quelques-unes des interrogations qu'elle suscite. Les questions soulevées, qu'il s'agisse des limites de la conception binaire de l'inégalité sélective 
ou encore des liens entre l'école et le peuplement d'un quartier, débouchent, dans certaines réflexions, sur une exigence de solidarité élargie.

\section{Les limites de la conception binaire de l'inégalité sélective}

Les zones d'éducation prioritaire ont été le dispositif par lequel a été introduite en France la notion d'inégalité sélective, réinterprétation de la discrimination positive pratiquée aux États-Unis d'abord en faveur des minorités ethniques (7). C'est, en effet, dès 1981 que le ministère Savary reconnaît la nécessité de créer des inégalités sélectives en faveur de zones socialement et culturellement défavorisées. Depuis lors cette mesure a connu un succès croissant jusqu'à ce que le rapport Moisan-Simon (1997) et les assises nationales des ZEP de 1998, destinées à la relance de l'éducation prioritaire, en soulignent la médiocre efficacité.

Les parents mobilisés en faveur du maintien en ZEP des écoles de leur quartier sont conduits à s'interroger quant à eux sur l'ajustement de la politique d'inégalité sélective à l'ampleur des problèmes rencontrés dans les quartiers en difficulté. En la matière, les propos des interviewés manifestent des degrés de sophistication variables qui vont du souci de préserver le dispositif en l'état pour les écoles de leur quartier à celui de repenser le dispositif ZEP, voire d'envisager des actions pédagogiques spécifiques. Tous sont amenés à prendre en compte le fait qu'il existe des endroits plus défavorisés, mais que la politique d'éducation prioritaire ne permet pas, en l'état, de faire face aux exigences éducatives de tels quartiers.

Du côté de l'Amicale, l'accent est mis, comme le rapporte l'un de ses représentants, sur l'existence de difficultés sociales réelles dans le quartier mais qui n'ont pas de retentissement au sein de l'école car les moyens alloués dans le cadre de la ZEP lui permettent précisément de faire face à la situation (voir infra: École et quartier : la menace du ghetto). Ce constat conduit une autre représentante à défendre l'idée selon laquelle les moyens alloués aux écoles des quartiers en grande difficulté sont insuffisants : "J'ai tout à fait conscience qu'il y a des quartiers qui ont des plus grandes difficultés que les nôtres et là il faut leur donner encore plus de moyens. Euh! je sais bien que c'est utopiste [...] C'est vrai que la question, je me la suis posée aussi au départ! Bon, mais après j'ai changé d'avis en me disant: ok, il y a pire ailleurs, mais c'est ailleurs qu'il faut faire autrement et nous, on nous laisse ça; nous ça marche, on nous laisse ça. » Si le souci de l'ailleurs apparaît ici directement connecté à celui de préserver un équilibre menacé qui la concerne directement, le propos suggère à la fois un cheminement de la réflexion et les difficultés de mise en œuvre de ce qui est suggéré.

(7) Pour une étude attentive des références américaines, voir notamment Damon (2000) et sur ce qui concerne la discrimination positive à l'école Meuret (2000). 
Du côté de la FCPE, la réflexion sur les limites de la conception binaire de la discrimination positive est plus nourrie. C'est ainsi que le représentant d'une des écoles maternelles, plutôt réservé au départ sur le bien-fondé de la mobilisation, critique avec force l'insuffisance des moyens octroyés dans les quartiers connaissant de très grandes difficultés : "Les problèmes sont tellement plus massifs à certains endroits que l'on se dit que ce n'est pas forcément les mêmes outils qu'il faut employer. C'est vrai que lorsqu'on travaille à moyens constants, les choses deviennent un peu difficiles [...] Est-ce que le quartier mérite d'être en ZEP ou ne mérite pas d'être en ZEP, je crois qu'il y a des raisons pour qu'il le soit et je crois que ce n'est pas scandaleux. Par contre c'est clair qu'il y a des endroits où il devrait y avoir des actions beaucoup plus fortes, des moyens beaucoup plus forts. » Pour ce représentant FCPE, qui pointe les contraintes liées à une refonte de la carte des ZEP à moyens constants, le dispositif tel qu'il est conçu est inadapté car il ne permet pas de traiter la complexité des niveaux de difficulté. Les propos de la représentante de l'Amicale attestent également que le constat de l'inadaptation de la politique des ZEP s'est constitué dans un va-et-vient entre une connaissance des écoles du quartier et des échos de situations plus éloignées : « À la limite, les gens lorsqu' on leur disait qu'en Seine-Saint-Denis, etc., ils disaient: "Bien oui, c'est pas parce qu'en Seine-Saint-Denis c'est beaucoup plus problématique, qu'ici il n'y a pas aussi des problèmes ; c'est en Seine-Saint-Denis qu'il faut mettre beaucoup plus de moyens plutôt que d'essayer de retirer les moyens ici."»

Un autre représentant FCPE, élu en élémentaire, évoque l'idée de «super ZEP », adaptée aux quartiers en grande difficulté et va jusqu'à décliner des mesures appropriées : «Ma réflexion serait de penser qu'il y a des nécessités dans les quartiers à hautes difficultés sociales, aujourd'hui, qui dépassent très largement cette discrimination particulière des ZEP. Ça va certainement beaucoup plus loin que les 25 élèves par classe, avec des enseignants formés de façon extrêmement précise, avec des décloisonnements pour qu'il y ait des petits groupes, avec la capacité de mettre un réseau d'aide sociale cohérent et que les choses ne soient pas simplement l'affaire de l'école seule et que, peutêtre, ce n'est pas la ZEP la solution. » Les suggestions faites sont nettement plus ambitieuses que ce que permet le dispositif ZEP et sa conclusion est réservée quant aux capacités de la politique d'éducation prioritaire à traiter le problème.

L'inspecteur de circonscription fait également ressortir les limites de la politique des ZEP, telle qu'elle est conçue, mais c'est pour mieux rappeler les difficultés que connaissent certaines autres écoles de la circonscription qu'il administre : " J'ai une circonscription qui est très variée avec des oppositions très nettes entre certaines écoles qui me paraissent être en très grosse difficulté, en particulier autour du quartier X... Et de temps en temps, je me dis que c'est pas normal que ces écoles-là aient exactement les mêmes moyens que [le quartier étudié]. En d'autres termes, je me demande si on fait des réseaux d'éducation prioritaire ou des zones d'éducation prioritaire, je me demande s'il ne devrait pas y avoir une hiérarchisation en fonction de la diffi- 
culté des écoles. »C'est d'abord son expérience quotidienne qui l'informe des limites de la politique d'inégalité sélective dans la mesure où elle conduit à octroyer les mêmes moyens à deux écoles dont les difficultés sont sans commune mesure. C'est en revanche la menace du retrait du statut ZEP des écoles où leurs enfants sont scolarisés qui incitent les représentants des parents à une réflexion sur le sujet. Tous posent, de façon convergente, la question de l'ajustement de la politique d'éducation prioritaire et des moyens engagés en fonction des difficultés rencontrées. Ce faisant, est suggérée une conception plus fine, plus graduée de la politique des ZEP où les moyens alloués sont proportionnels aux difficultés rencontrées avec, dans les cas les plus problématiques, le déblocage de ressources importantes et la mise en place de dispositifs innovants de traitement des problèmes. La prise en compte d'une gradation des niveaux de difficulté scolaire atteste d'une exigence de solidarité dont l'espace de référence est élargi bien au-delà du seul voisinage.

\section{École et quartier : la menace du ghetto}

Beaucoup de travaux ont aujourd'hui mis en évidence les migrations des élèves et l'évitement des établissements publics des quartiers défavorisés, réputés «sensibles » ou «difficiles », par les familles des classes moyennes (Broccolichi et van Zanten, 1997 ; van Zanten, 2001). Ces déplacements se font d'une école publique vers une autre, lorsque c'est possible et, plus encore, du public vers le privé. La rigidification de la carte scolaire peut aussi, dans certains lieux, avoir des effets sur les migrations résidentielles (et pas seulement scolaires) et générer à terme la constitution de véritables ghettos urbains dans lesquels les phénomènes d'évasion scolaire ne se posent plus en raison de l'absence totale de mixité sociale. Non seulement l'école est en interaction avec son environnement social et urbain, mais de plus en plus elle contribue à en dessiner la physionomie. La préservation d'une mixité sociale, indissociablement urbaine et scolaire, est apparue, non sans ambiguïté, comme un problème central soulevé par la mobilisation étudiée.

C'est ainsi qu'un des membres de l'Amicale s'interroge sur le dilemme que provoquerait chez lui le délabrement social et scolaire de l'école de secteur : «Un des enjeux forts, c'était le maintien de la mixité, la ZEP était aussi la garantie de cette mixité [...] Si l'école fonctionne bien, on ne se pose pas la question, du moins dans mon cas, je ne me pose pas la question de la composition sociale du quartier, le fait de savoir s'il y a des enfants en difficulté ou pas dans l'école. Ce qui m'intéresse, c'est que l'école ait les moyens de répondre à cette situation. C'est vrai que, quelles que soient nos convictions par ailleurs, si mon fils était tombé dans une école complètement dégradée, avec des enseignants qui n'ont pas les moyens, qui sont dépassés par la situation, je me serais posé la question de le maintenir dans cette école. » L'évocation de l'évasion scolaire a pu parfois apparaître comme une menace, voire comme un chantage brandi par des parents de milieux favorisés. C'est en 
partie l'interprétation de l'inspecteur de circonscription: "Les parents que j'ai rencontrés étaient globalement issus de couches relativement favorisées et l'argument qu'ils tenaient, c'était: "Nous laissons nos enfants parce que l'école est en ZEP [...] parce qu'elle reçoit un certain nombre de moyens qui font que les difficultés peuvent être traitées. Si vous l'enlevez de la ZEP, eh bien on va perdre ces moyens. Donc ça va faire une école qui va avoir tendance à se ghettoïser et nous ne resterons pas."»

Cependant l'inspecteur, tout en manifestant un soupçon d'agacement, se trouve démuni face à un tel discours : il n'a aucun moyen de contraindre les familles qui y ont recours à continuer à vivre dans un quartier qui leur fait craindre pour la scolarité et l'avenir de leurs enfants. Il évoque d'ailleurs luimême, lorsqu'il parle de la sociographie de sa circonscription, les déménagements des familles de classes moyennes au moment de l'entrée dans le secondaire et le départ des familles d'origine européenne d'une des écoles élémentaires qu'il administre. Loin d'être isolé, le phénomène de migration scolaire s'étend également aux familles d'origine immigrée comme en témoigne le cas d'une adhérente de l'Amicale qui, quoique de culture musulmane, a envoyé son fils aîné dans un collège catholique pour le prémunir de fréquentations douteuses : "On ne pensait pas systématiquement à l'école privée, ma démarche, c'était changer uniquement de secteur. Comme ça n'a rien donné - la dérogation a été refusée - donc on a atterri dans le privé. Donc, dans le privé, je ne me sens pas du tout... D'abord c'est un collège catho [...] Ces parents-là sont trop différents de moi dans les façons de voir les choses [...] J'aurais souhaité que mon fils soit dans le public. » Faisant tout à la fois le sacrifice de ses convictions et de son investissement de parent d'élève, l'interviewée ressent comme une profonde injustice la rigidité de la carte scolaire, parce qu'elle est indexée sur une géographie qui n'est pas neutre socialement. «Ce que je trouve dommage c'est que d'un arrondissement à un autre, on n'est vraiment pas logé à la même enseigne, on a tel collège, point final. On ne peut pas bouger de là, qu'il soit bon, qu'il soit mauvais, on n'a pas le choix. Je trouve que c'est vraiment une injustice. Si on pouvait avoir des collèges un peu hétérogènes, un petit peu partout, sans qu'il $\mathrm{y}$ ait des ghettos ou des collèges élitistes, c'est l'idéal. »

L'idéal de l'établissement hétérogène auquel aspire cette mère de famille est assez partagé par les parents qui se sont mobilisés pour préserver le statut ZEP de leurs écoles. Les personnes rencontrées, adhérentes d'associations de parents d'élèves, ont manifesté leur attachement au quartier tel qu'il est, à son caractère populaire, au mélange des populations qui s'y côtoient. Leur fort investissement dans l'école, que montrent les différents propos rapportés, en font un point névralgique. L'idéal d'école républicaine, socialement et culturellement mixte, auxquels ils souscrivent en raison de leurs convictions politiques et laïques tant du côté de la FCPE que de l'Amicale, leur semble bien incarné dans l'école du quartier, mais sous un équilibre très fragile : " pas un quartier hyper à problèmes mais quand même un quartier qui est un peu sur le fil du rasoir ». Grâce aux moyens supplémentaires offerts par le statut ZEP, ils ont l'impression de contribuer à cette mixité : «S'il n'y a pas de problèmes, 
c'est peut-être parce que des gens comme nous laissons nos enfants dans ces écoles ; pourquoi on laisse nos enfants dans les écoles publiques du quartier? C'est peut-être justement parce qu'il y a aussi des moyens. »

Se trouve ainsi mise au jour, sans nuance ni précaution oratoire, l'ambiguïté des objectifs de mixité sociale auxquels les politiques éducatives ne cessent de se référer sans jamais les afficher explicitement comme priorité. Dans les quartiers à dominante populaire, il n'est jamais possible d'être certain que les familles de classes moyennes qui y vivent continuent d'inscrire leurs enfants à l'école publique. Contribuant à la mixité sociale de l'école et du quartier, elles sont aussi celles qui, en obtenant des dérogations à la carte scolaire, en migrant vers l'école privée ou en changeant de lieu de résidence peuvent, en peu de temps, transformer l'école républicaine, en laquelle elles sont les premières à croire, en ghetto social, voire à faire d'un quartier culturellement et socialement mixte un ghetto urbain. L'administration de l'Éducation nationale est impuissante face à ce risque dans la mesure où elle n'a pas la maîtrise de tous les paramètres : quand bien même elle chercherait à rendre la carte scolaire plus stricte, seul paramètre qui soit partiellement de son ressort, une telle mesure peut faire fuir ces familles vers l'école privée ou encore les faire déménager vers d'autres quartiers. Un des inspecteurs interrogés, responsable d'une autre circonscription parisienne et qui a précédemment occupé un poste dans un environnement très difficile, fait état du problème. Son expérience le conduit à considérer que la rentabilité de l'investissement éducatif est un élément à retenir dans la politique des ZEP. Elle ne devrait pas tant consister à donner davantage à ceux qui auraient moins qu'à donner davantage aux écoles qui peuvent le mieux en profiter : «Les indicateurs sont bons pour tout ce qui est réussite scolaire, alors je dirais que, pour avoir travaillé à la Grande Borne et pouvoir comparer les choses, bien entendu ça n'a rien à voir avec la Grande Borne, il faut quand même être parfaitement clair, justement ce sont des secteurs sur lesquels quand on travaille, comme la dégradation n'est pas continue, le travail qu'on fournit porte ses fruits. »

Les parents des classes moyennes sont incités, dans une telle conception, à entrer dans un raisonnement utilitariste en maintenant leurs enfants dans les écoles de secteur, lorsqu'ils y habitent, voire à vaincre leurs éventuelles réticences de mobilité résidentielle vers ce type de quartier, en raison des avantages offerts par le classement en ZEP : effectifs d'élèves moins chargés, moyens pédagogiques supplémentaires, projets innovants, enseignants motivés. Plutôt que de recourir à la dérogation pour une autre école publique, à l'inscription dans une école privée ou au déménagement, ils seraient encouragés à contribuer à la réussite du dispositif d'éducation prioritaire. C'est la question du déplacement de la politique d'inégalité sélective en matière éducative qui est alors posée. D'un seul objectif d'aide aux plus défavorisés, elle basculerait vers un objectif de mixité sociale; d'un objectif strictement éducatif, elle évoluerait vers un objectif d'aménagement du territoire, conçu comme mieux apte à contribuer à la réduction des inégalités. Loin d'être absente, une exigence de solidarité élargie irrigue une telle conception, qui 
suggère la mise en synergie des politiques publiques et qui met l'accent sur la nécessité d'organiser la mixité sociale.

\section{Extension du débat, clôture de la controverse et rapport de force}

La dynamique de la controverse conduit les parents à élaborer un argumentaire publiquement recevable qui cherche à rendre compatibles la réalité des écoles que fréquentent leurs enfants, les caractéristiques du quartier dans lequel ils vivent avec la prise en compte d'une gamme étendue d'autres situations, des plus favorisées aux plus défavorisées. Ce faisant, ils sont conduits à ré-inventer la politique qu'ils critiquent. Jusqu'où parviennent-ils à faire entendre le bien-fondé de leur position et de leurs propositions ? Dans quelle mesure l'administration de l'Éducation nationale se montre-t-elle ou pas réceptive aux arguments énoncés ? L'élaboration argumentative tient-elle, enfin, une place dans la clôture de la controverse ou celle-ci cède-t-elle le pas à des rapports de force ?

La mobilisation étudiée a rapidement fédéré les deux associations de parents, les parents militants et les parents non affiliés, les parents et les équipes pédagogiques, les écoles maternelles et les écoles élémentaires, les écoles du quartier et celles d'autres quartiers concernés par une sortie du dispositif ZEP. L'engagement de nouveaux acteurs, toujours plus divers, contribue à élargir le débat, à arracher celui-ci du seul ancrage de proximité et permet de tenir à distance l'accusation selon laquelle la mobilisation serait exclusivement tournée vers des intérêts égoïstes. C'est un souci identique qui conduit les associations à alerter les élus politiques comme en témoigne ce représentant de l'Amicale : "Nous avons envoyé des lettres à tous les élus, à la mairie de Paris, au maire du $\mathrm{XX}^{\mathrm{e}}$ arrondissement, aux groupes politiques de la mairie du $\mathrm{XX}^{\mathrm{e}}$ arrondissement pour les alerter de la situation et les inviter à nos réunions. Donc, on a eu très vite un soutien sans borne du Parti communiste et des Verts qui se sont mobilisés, qui nous ont offert leurs moyens, qui se sont mis à notre disposition pour tirer des tracts. » Mais étendre la mobilisation par l'enrôlement d'acteurs à la fois plus nombreux et aptes à la relayer nécessite un travail argumentatif approprié qui atteste de la généralité de la cause soutenue. Comme la recherche d'un argumentaire recevable, qui fait l'objet de réunions de travail entre parents et enseignants, ce type d'élargissement témoigne d'un engagement dans une logique tactique-stratégique (Corcuff et Sanier, 2000 ; Lafaye, 1994) qui autorise la construction d'un rapport de force favorable.

$\mathrm{Si}$, pour le conseil local de la FCPE, cet élargissement va de soi, celui-ci est objet d'un débat interne à l'Amicale : "Chez certains parents de l'Amicale, il y avait quand même l'idée qu'il fallait d'abord défendre notre bout de gras et qu'après tous les autres n'avaient qu'à se bouger. Mais ça n'a pas duré très longtemps parce que, très vite, on s'est aperçu que l'enjeu n'était pas foncièrement local, que c'était un enjeu au moins parisien sinon national 
pour la politique de relance des ZEP. » La tentation égoïste, illustrée ici par la métaphore dénonciatoire du « bout de gras », est écartée au profit d'une mobilisation conçue comme une contribution au débat général sur l'école, plus particulièrement à la politique de relance des ZEP. Le même adhérent de l'Amicale dit par ailleurs : " Il y avait une philosophie de l'éducation prioritaire que nous voulions défendre. » Le cas local est explicitement conçu comme permettant de mieux éprouver l'ajustement d'une politique publique générale : "Nous avons réussi à solliciter une question orale au Sénat par l'intermédiaire d'un élu communiste du $\mathrm{XX}^{\mathrm{e}}$. Il a contacté un élu du groupe communiste du Sénat qui a posé une question orale à Ségolène Royal, laquelle portait sur le groupe scolaire, puisqu'à cette époque, c'était considéré un peu comme un symbole, en tout cas pour Paris, de la manière dont s'opérait cette relance [des ZEP]. » (8).

Mais on arrive aux confins du régime de la justification publique, lorsque les acteurs eux-mêmes évoquent la nécessité de créer un rapport de force favorable vis-à-vis de l'administration scolaire : «On était quand même assez convaincus qu'en faisant bouger un peu les journaux ça avait de toute façon plus de poids que les délégations qu'on pouvait faire, les rassemblements dans la rue. De toute façon, nous n'avions pas les forces, les moyens, d'engager un rapport de force de ce côté-là. Donc c'était un rapport de force en termes de débat public qu'on pouvait faire. » Chez cet adhérent de l'Amicale, la notion de débat public - qui signifie qu'un problème est mis en discussion dans l'arène publique, ici par l'intermédiaire des médias - est articulée à celle de rapport de force. Or, la force et les rapports de force sont généralement pensés comme étrangers au régime de justification publique, ce qui rend nécessaire l'examen des modalités de leur accrochage (Thévenot, 1996 ; Nordacchione, 2003). En décomposant le récit, on constate que la campagne de presse et la couverture médiatique qui l'accompagne sont décrites comme étayées par un dossier argumenté : "On a fait une conférence de presse, dans le cadre du quartier, pour laquelle on avait constitué un dossier un peu conséquent; on avait fait tout un argumentaire. » Or, la suite du récit montre que le bienfondé des arguments importe moins, pour sensibiliser l'administration scolaire, que la pression médiatique qui les relaie. Les représentants des services académiques estiment d'ailleurs, lorsqu'on les interroge, que les parents ont développé une mobilisation en cherchant à construire un rapport de force favorable, ce dont ils admettent la pertinence puisque, comme l'explique l'inspecteur de la circonscription, ce sont ceux qui se mobilisent le plus qui sortent gagnants.

La recherche d'arguments fondés, avec l'objectif de construire une cause publique détachée de la défense d'intérêts particuliers, a davantage pour effet de souder le mouvement, de donner de la force à ceux qui le mènent, grâce à l'élaboration de revendications légitimes, que de convaincre l'administration.

(8) La manière dont a été pensée la mobilisation - comme contribution au débat général sur l'école - a eu des effets, aux dires de cet interviewé, sur la conception même de l'Amicale en favorisant, au niveau interne, la remise en cause de son caractère apolitique. 
Cette recherche d'une argumentation recevable se double d'une démarche tactique-stratégique qu'une directrice d'école maternelle explicite. Il y a les arguments (qui, s'ils n'ont pas été déterminants pour faire pencher l'administration, ont constitué un horizon de légitimité permettant la prise de conscience des gens et la constitution du mouvement). Il y a la mobilisation (conçue comme un moyen de pression sur l'administration de l'Éducation nationale). De plus, l'élaboration d'arguments acceptables assure la crédibilité et la légitimité de la mobilisation aux yeux des politiques comme des médias. Le relais que ces derniers permettent, par leur inscription dans des réseaux longs, et qui en définitive contribue à faire basculer l'administration, ne doit pas laisser croire que la recherche argumentaire a été superflue. Elle est une condition essentielle sinon première, celle qui permet au mouvement de se constituer, de grossir, d'être entendu et relayé : elle met en branle le mouvement, lui donne sa légitimité et permet son succès quand bien même les arguments tendent à s'effacer devant la force dont ils sont le moteur.

Ce constat conduit plusieurs acteurs à se plaindre d'une sortie du conflit par la force et à regretter que leurs arguments n'aient pas été pris en compte, ni même entendus : "C'est le rapport de force qui nous a fait gagner, c'est pas nos arguments, ils n'ont pas été écoutés. » Cette déception diffuse, plus marquée chez les parents que chez les enseignants, n'est-elle pas due au fait que l'objet de la mobilisation s'est progressivement déplacé du seul maintien en ZEP des écoles du quartier vers l'aspiration, plus globale et plus exigeante, de contribuer à une politique de relance des ZEP largement ignorée des interlocuteurs administratifs?

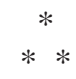

Le premier intérêt de l'étude de cas présentée est d'offrir une grille de lecture d'une forme d'action collective qui constitue à la fois l'aiguillon et l'avatar de la relocalisation des politiques publiques nationales. De la même façon que s'est substituée à l'approche dite top down (i.e. par le haut) des politiques publiques une approche dite bottom up (i.e. par le bas et à travers l'expérimentation), l'action collective organisée sur le modèle du parti politique, du syndicat ou des grandes fédérations associatives (pendants de la verticalité étatique) a laissé la place à des formes localisées et émergentes d'action revendicative. Notre analyse de la mobilisation des parents contre le déclassement du dispositif ZEP du groupe d'écoles où leurs enfants sont scolarisés montre cependant que de telles formes d'action ne se réduisent pas à des revendications limitées aux seuls intérêts particuliers, du type de celles que l'on qualifie, dans le domaine de l'aménagement, de NIMBY. Les formes d'action que nous avons étudiées sont à la fois «localisées » et «émergentes », dans la mesure où elles sont susceptibles de faire émerger et de poser des questions d'intérêt général à partir d'un ancrage localisé, retrouvant, mutatis mutandis, les racines des mouvements collectifs. On a pu voir, 
ce faisant, que les lignes de fracture usuelles entre associations locales et fédérations nationales tendent à s'effacer.

La mobilisation analysée montre la possibilité pour des mouvements locaux de construire du général, de trouver des points d'articulation avec des revendications non particularistes, sans pour autant perdre de vue les motifs initiaux d'insatisfaction. C'est parce que pèsent sur l'action revendicative de fortes contraintes de généralité et de recevabilité des arguments échangés que des mobilisations à première vue locales cessent de l'être dans le mouvement même de publicité de leurs revendications. Ce faisant, les motifs de l'action sont désubjectivés, rendus partiellement extérieurs aux personnes et déposés dans une grammaire publiquement disponible (Trom, 2001).

La clôture de la controverse atteste, enfin, de la perméabilité entre deux formes d'engagement des personnes, généralement pensées comme antinomiques : un engagement dans un régime de justification publique, qui permet à la mobilisation de se constituer en cause publiquement recevable et ainsi de rassembler autour d'elle de plus en plus de monde, et un engagement dans le rapport de force. Dans ce dernier cas, les arguments cèdent la place à la force, entendue comme la somme des acteurs qui les mobilisent, les relaient ou peuvent être atteints par eux (les lecteurs des journaux). C'est la force qui s'impose à l'administration parisienne et la conduit à abandonner le projet contesté. L'administration accède à la satisfaction de la revendication des parents, mais méconnaît sa légitimité. La réponse qu'elle propose déconsidère les efforts des parents d'élèves dont l'action est assimilée à la seule construction d'un rapport de force dont le juste est absent. D'une part, elle freine les possibilités réelles de prise au sérieux des mouvements localisés qui constituent pourtant aujourd'hui une forme de plus en plus répandue d'action collective, s'interdisant de prévenir son caractère imprévisible par la mise en œuvre d'un type de relations approprié. D'autre part, elle ferme le débat public ouvert par la mobilisation, s'empêchant elle-même de participer plus étroitement à la redéfinition de la politique d'éducation prioritaire.

Yves DUTERCQ

Groupes d'Études Sociologiques - INRP

29, rue d'Ulm - 75005 Paris

y.dutercq@wanadoo.fr

Claudette LAFAYE

Groupe de Sociologie Politique et Morale - EHESS-CNRS 10, rue Monsieur le Prince - 75006 Paris

lafaye@ehess.fr 


\section{RÉFÉRENCES BIBLIOGRAPHIQUES}

Ashworth A., 1991. - «L'usager face à l'école : l'émergence de l'individu », Savoir, 3.

Ballion R., 1982. - Les consommateurs d'école. Stratégies éducatives des familles, Paris, Stock.

Barthélémy M., 1995. - «Des militants de l'école : les associations de parents d'élèves en France », Revue française de sociologie, 36, 3, pp. 439-472.

Boltanski L., 1990. - L'amour et la justice comme compétences, Paris, Métailié.

Boltanski L., Thévenot L. (éds.), 1989. - Justesse et justice dans le travail, Paris, Presses Universitaires de France (Cahiers du Centre d'Études de l'Emploi).

- 1991. - De la justification. Les économies de la grandeur, Paris, Gallimard.

Broccolichi S., van Zanten A., 1997. - «Espaces de concurrence et circuits de scolarisation. L'évitement des collèges publics d'un district de la banlieue parisienne », Annales de la recherche urbaine, 75 , pp. 5-31.

Céfaï D., Lafaye C., 2001. - «Lieux et moments d'une mobilisation collective. Le cas d'une association de quartier» dans D. Céfaï, D. Trom (dirs.), Les formes de l'action collective. Mobilisations dans des arènes publiques, Paris, EHESS (Raisons pratiques, 12).

Chauvière M., Godbout J. T. (éds.), 1992. - Les usagers entre marché et citoyenneté, Paris, L'Harmattan.

Corcuff P., Sanier M., 2000. - « Politique publique et action stratégique en contexte de décentralisation. Aperçus d'un processus décisionnel après la bataille », Annales histoire, science sociale, 4.

Damon J., 2000. - Discrimination positive et affirmative action, Paris, CNAF-Bureau de la Recherche (Dossier d'étude, 7).

Derouet J.-L., 1991. - «Décentralisation et droits des usagers », Savoir, 3-4, pp. 619-641.

- 1992. - École et justice, Paris, Métailié.

Les dossiers d'Éducation et formations, 1998. - « Les familles et l'école. Apports de la recherche, points de vue de praticiens », Paris, Ministère de l'Éducation nationale, DPD, 101.

Desrosières A., 1989. - «L'opposition entre deux formes d'enquête : monographie et statistiques » dans L. Boltanski, L. Thévenot (éds.), Justesse et justice dans le travail, Paris, Presses Universitaires de France (Cahiers du Centre d'Études de l'Emploi), pp. 1-10.

- 1993a. - La politique des grands nombres, Paris, La Découverte.

— 1993b. - « Le territoire et la localité : deux langages statistiques », Paris, INSEE, note 13 janvier, $n^{0} 3 / \mathrm{G} 305$.

Dubet F. (dir.), 1997. - École, familles : le malentendu, Paris, Textuel.

Dutercq Y., 1993. - Consommateurs d'école et modernisation du service public. Les conflits entre parents d'élèves et établissements scolaires peuvent-ils se construire en cause collective ? Paris, Commissariat Général du Plan.

— 1995. - «Une partie inégale. Les interventions publiques des parents d'élèves », Politix, 31.

- 1998. - «La résistible ascension de la cause des parents d'élèves en France » dans M. Hardy et al. (éds.), L'école et les changements sociaux : défi à la sociologie? Montréal, Logiques.

- 2000. - Politiques éducatives et évaluation. Querelles de territoires, Paris, Presses Universitaires de France.

- 2001. - «Les parents d'élèves entre absence et consommation », Revue française de pédagogie, 134, pp. 111-122.

Goffman E., 1991. - Les cadres de l'expérience, Paris, Éditions de Minuit.

Ion J., 1999. - «Engagements associatifs et espaces publics », Mouvements, 3.

Fourniau J.-M., 1996. - «Transparence des décisions et participation des citoyens », Techniques, territoires et sociétés, 31. 
Jeannot G., 1998. - Les usagers du service public, Paris, Presses Universitaires de France (Que-saisje? 3359).

Lafaye C., 1990. - «Situations tendues et sens ordinaires de la justice dans une administration municipale », Revue française de sociologie, 31, 2, pp. 199-223.

- 1994. - «Aménager un site littoral. Entre politique et pragmatisme », Études rurales, 133-134, pp. $163-180$.

Lepetit B., 1995. - «Le présent de l'histoire » dans B. Lepetit (éd.), Les formes de l'expérience. Une autre histoire sociale, Paris, Albin Michel.

Lolive J., 1999. - Les contestations du TGV Méditerranée, Paris, L'Harmattan (Logique politique).

Masson Ph., 1997. - «Élèves, parents d'élèves et agents scolaires dans le processus d'orientation », Revue française de sociologie, 38, 1, pp. 119-142.

Meuret D., 2000. - «Les politiques de discrimination positive en France et à l'étranger » dans A. van Zanten, L'école. L'état des savoirs, Paris, La Découverte, pp. 112-120.

Moisan C., Simon J., 1997. - Les déterminants de la réussite scolaire dans les zones d'éducation prioritaire, Paris, INRP.

Nordacchione G., 2003. - Contester en infériorité. Les mobilisations de quartier en Argentine, de Menem à nos jours (1990-2002), Paris, Centre d'Étude des Mouvements Sociaux, EHESS [à paraître].

Padioleau J. G., 1993. - «L'action publique : du substantialisme au pragmatisme », Techniques, territoires et sociétés, 22-23.

Ricoeur P., 1983. - Temps et récit, tome 1, Paris, Le Seuil (Point-Seuil).

Thévenot L., 1996. - «Stratégie, intérêts et justifications. À propos d'une comparaison FranceÉtats-Unis des conflits d'aménagement », Techniques, territoires et sociétés, 31.

- 1999. - «Faire entendre une voix, régimes d'engagement dans les mouvements sociaux», Mouvements, 3.

Trom D., 1999. - « De la réfutation de l'effet $N I M B Y$ considérée comme une pratique militante. Notes pour une approche pragmatique de l'activité revendicative », Revue française de science politique, $49,1$.

- 2001. - «Grammaires de la mobilisation et vocabulaire de motif» dans D. Céfaï, D. Trom (dirs.), Les formes de l'action collective. Mobilisations dans des arènes publiques, Paris, EHESS (Raisons pratiques, 12).

Van Zanten A., 2001. - L'école de la périphérie, Paris, Presses Universitaires de France.

Warin Ph. (dir.), 1997. - Quelle modernisation des services publics? Les usagers au cœur des réformes, Paris, La Découverte. 